\title{
Construction of Classroom Interactive System of College and the Research of Application Model
}

\author{
Jianzhan Zhang, Guobiao Liang, Rong Jiang \\ Office academic of affairs, Hunan University, China \\ *joechi@hnu.edu.cn
}

Keywords: College Classroom; Interactive System; Model

\begin{abstract}
This research analyzes teachers and students' feedback to interaction and knowledge in the classroom, and designing the classroom interaction system. That based on investigating the classroom interaction system of domestic and foreign, combined with the practical application of classroom teachers and students questionnaire. The whole system model is divided into application module, decision module and management module. These modules could transfer the data each other and analyze the data. The teachers, students and management decision-makers of college can use this system to know teachers' and students' feedback about knowledge, to control learning discipline, to static students' daily performance and teaching evaluation.
\end{abstract}

\section{Background and contents}

The form of college class is usually "one-many", one teacher and many students. This form is called "big class teaching", one teacher teaches hundreds of students. It is a very big challenge for a teacher to make so many students focus on course. It is a problem for a student to make the teacher understand her or his own learning situation and get real-time guidance. The interaction between teacher and student, also student and student is limited in this traditional classroom. Interaction and feedback between teacher and student usually lag behind, and interactive effect is not very good.

The main content of this study is to solve above problems and difficulties. In ordered to help colleges use information technology to teach and manage. This research focus on the technology theory applied in classroom teaching process, combining with the theory of teaching design and teaching evaluation, to explore that college teachers how to stimulate learning enthusiasm of students, and how to build appropriate teaching rhythm to attract students' attention and active classroom atmosphere. To collect information about students so as to determine the teaching direction, to assess and understand students' learning.

\section{Classroom interaction system research at home and abroad}

In the $1990 \mathrm{~s}$,Eric mazur who is the professor of department of physics, Harvard University, proposed peer teaching method, that uses the test about concepts which are designed to reveal the students' misconcepts and guided students to step explore, With the help of computer systems or interactive response option card to guide students to participate in the teaching process, change the traditional teaching method to an independent study and cooperative inquiry which is based on analysis of the concept, to build an innovative teaching mode which is filled with cooperative learning, student interaction, teacher-student interaction in classroom.

The emergence of education technology has impacted teaching method that only chalk and blackboard were used in class, with the development of computer network; expand the scope of 
learning resources and school education. In recent years, the using of classroom response systems, CRS, brings new ideas, methods and tools for the teaching model reform and teaching research. The successful application of famous colleges in the United States, such as Harvard and MIT, CRS received the affirmation of education, has been widely applied in foreign colleges [1-2].

\begin{tabular}{|c|c|c|}
\hline $\begin{array}{c}2012-2013 \text { Global } \\
\text { university rankings }\end{array}$ & School Name & Country \\
\hline 1 & California Institute of Technology & USA \\
\hline 2 & Stanford University & USA \\
\hline 3 & Oxford University & US \\
\hline 4 & Harvard University & USA \\
\hline 5 & Princeton University & USA \\
\hline 6 & Cambridge & US \\
\hline 7 & Imperial College London & US \\
\hline 8 & University of California, Berkeley & USA \\
\hline 9 & University of Chicago & USA \\
\hline 10 & $\ldots . .$. & $\ldots \ldots$ \\
\hline$\ldots \ldots$ & & $\ldots .$. \\
\hline
\end{tabular}

Table 1 . The Times ranking in 2013

In our country, the large-scale study about the classroom interaction system is less. National Taiwan University has used this system to help teachers to increase the chance of class interaction, and to improve teaching effect. This system make teachers get feedback instantly. Teachers can know students, learning progress, then to adjust their teaching progress and teaching method. Peking University, Central China Normal University, Sichuan University and other colleges are starting to use this system in teaching [3-5].

\section{The system user demand survey and related data mining}

In this study, this system is called classroom interactive system, to research how to raise interaction.

Teachers' requirements: through random testing in the teaching process, the system can collect information about students' learning. This progress is very rapid, simple, and synchronous. Such as: check the test results and analysis report, the teaching effect evaluation results, students' attendance, real-time class students for feedback on the situation of students' knowledge of information. Teachers use this information to decide whether to improve the teaching design. Two choices were provided for teachers before class and after class, there are paper test and no paper test. No paper text is that teachers upload papers to the system with their permission. On the one hand, they can grasp the learning situation of the students; On the other hand, they can also give certain common scores according to the test results. This system also provides historical achievement inquiry to teachers to know how their students learn for a period of time[5].

Students' requirements: The system can submit learning information about all of the students, to know its real-time learning effect that can make them pay attention to learning. Such as: check attendance, test results, test reports, etc. Any selectivity question, students just need press their mini keyboard to answer. All of answers are delivered to the server, which calculates the answers of the students' feedback to the teacher's computer screen. The result is usually a bar chart or a pie chart. Students can know whether they progress or not by searching historical achievement.

Administrators' (teaching secretary, counselor, etc.) requirements: they can know the conversation and learning effect about students through the system. 
Decision makers' (principal, dean, etc.) requirements: they can know teaching effect and teaching quality of teachers, evaluate teachers' work performance.

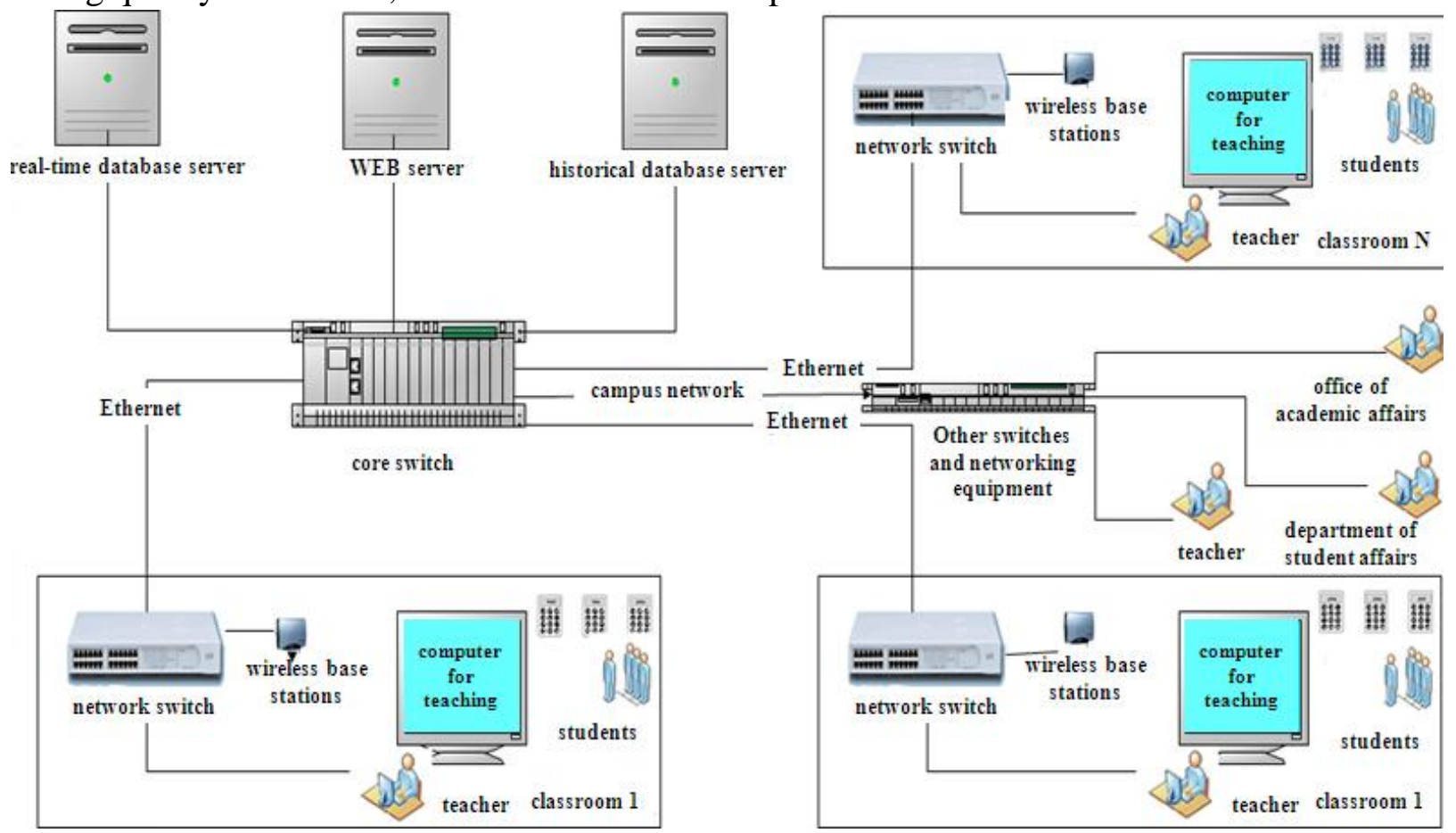

Figure 1. Construction of classroom interactive system of college

Classroom interactive system of college includes student terminal equipment, data acquisition stations, data server, management and teachers' personal devices.

Terminal equipment for student. Terminal equipment for student has a unique ID with the students' basic information. wireless frequency hopping communication is applied in it, to be compatible with ISO/IEC 18000-4 standard data protocol to interact with the base station, download the information such as classroom configuration, the questions about the topics, and upload the basic information and problem solving about the subject. The device also has intelligent display and keyboard. It is convenient for students to complete the corresponding operations.

Base station equipment. Base station equipment is installed in all of classrooms. This device, on the one hand, can complete information exchange by wireless frequency hopping communication with student terminal equipment; on the other hand, can be controlled by server through connecting TCP/IP protocol with the data server, to automatically deploy program for teaching plans and questions. And submit the information of check-in and answer from terminal to the server.

The servers. The servers include real-time database server, historical database server and WEB server. Real-time database server used to store the real-time interactive data and evaluating platform running data, it runs with real-time data acquisition server to guarantee the performance of the system; historical database server used to copy historical data from the backup platform to guarantee the security of data; WEB server provides software support for teaching plans deign and download, real-time interactive courseware, rapid assessment analysis of the teaching effect assessment analysis software support, and provides the report generation and printing services.

Personal computer for teachers and management. Personal computers for teachers and management connect to the server via the internet to carry out works about teaching plans deign, real-time interactive class feedback, evaluation of teaching effect, and call the relevant data and generate reports according to demand. 
Importing teaching plan and system configuration data. Teaching managers can use pre-defined standard formats to import teaching plan to generate configuration data of curriculum, which is need for base station in each classroom, pass relevant data through campus network, and automatically configure the base station to the data server.

Information management of Student Attendance. Students to complete the check-in by using a handheld terminal registered to the respective base stations. The data server collects students' information to teaching managers who can use it to control the rate of students' attendance Rate.

Real-time interactive feedback in class. The instructor can use personal computers to access the data server, to obtain students' information in class, and to deign course questions by using interactive teaching software, course discussion and rapid test. To improve teaching effect through the teaching interaction, and timely grasp how the students learn, adjust teaching pace and focus of teaching.

Rapid assessment teaching effect. Teachers, teaching managers can only use this platform to test and evaluate students and teachers without paper, and realize the automatic evaluation and score statistics. Teaching managers can also organize students to evaluate their teachers with this system.

\section{Benefit analysis}

Students can be back to the classroom because of student attendance; Teachers can use immediate and efficient test to receive timely feedback, and the students can make an accurate assessment to their teachers. so as to enhance teaching effectiveness. Traditional teaching assessments are very few, and not be quantified. This system can get a lot of information, which is benefit to enhance the capabilities of teaching evaluation. Teaching management staff can effectively monitor student attendance, track learning progress, to enhance the management level.

\section{References}

[1] Ping Zhang, ERIC MAZUR, Peer-Instruction-The new teaching method from Harvard University physics curriculum, J. China University Teaching. 8 (2010) 69-71.

[2] Mazur E. Peer, Instruction:A User'S Manual, NJ: PrenticeHall, UpperSaddle River,(1997).

[3] Shengfeng Li, Hong Cui, Lisha Cheng, The application of Clicker in the interactive teaching, J. China modern educational equipment. 20(2010)19-20.

[4] Xiaoe Dai, The form and features that intelligent feedback system participates in the interactive classroom of college, J. China audio-visual education. 304(2012)111.

[5] Yaqiang Cui, Chunyan Wang, Qi Yu, The application of interactive classroom teaching system in colleges and universities teaching, J. The application of interactive classroom teaching system in colleges and universities teaching. Distance education in China. 5(2013)70. 